\title{
Thoracic Enhanced Recovery After Surgery: Single Academic Center Observations After Implementation
}

\author{
Audrey L. Khoury, MD, MPH, Lavinia M. Kolarczyk, MD, Paula D. Strassle, PhD, \\ Cynthia Feltner, MD, MPH, Lyla M. Hance, MPH, Emily G. Teeter, MD, \\ Benjamin E. Haithcock, MD, and Jason M. Long, MD, MPH
}

UNC School of Medicine, University of North Carolina at Chapel Hill, Chapel Hill, North Carolina; Gillings School of Global Public Health, University of North Carolina at Chapel Hill, Chapel Hill, North Carolina; Departments of Surgery, Anesthesiology, Epidemiology, and Medicine, UNC School of Medicine, University of North Carolina at Chapel Hill, Chapel Hill, North Carolina; and Cecil G. Sheps Center for Health Services Research, University of North Carolina at Chapel Hill, Chapel Hill, North Carolina

Background. Enhanced recovery after surgery (ERAS) is an evidence-based, multidisciplinary perioperative care model shown to reduce complications and hospital length of stay (LOS). While some thoracic ERAS studies were inconclusive, others demonstrated that ERAS improves patient outcomes after lung resections and provides more cost-effective care. We aimed to investigate the effects of preliminary implementation of an ERAS protocol, in comparison with conventional care, on lung resection outcomes at a single academic institution.

Methods. In this observational study, adult patients undergoing lung resections during the pre-ERAS (April 2014 to September 2015) and post-ERAS (January 2016 to May 2017) periods were identified. Relevant demographic, preoperative, anesthesia, and surgical variables were collected. Pre-ERAS and post-ERAS cohorts were compared in terms of hospital LOS, postoperative complications, and 30-day outcomes.

Results. We identified 264 patients, half in each cohort. Pre-ERAS and post-ERAS groups were similar with

$I^{n}$ n the 1990s, Henrik Kehlet developed the concept of enhanced recovery after surgery (ERAS), an evidencebased, multidisciplinary perioperative care model. This model has been shown to reduce complications and hospital length of stay (LOS). ${ }^{1,2}$ The 3 phases of a thoracic ERAS pathway are as follows: the preoperative phase includes counseling, nutrition, and optimization of comorbidities; the intraoperative phase incorporates multimodal analgesic medications and techniques (including regional anesthesia with epidural and/or intercostal block [ICB]), limiting crystalloids, and minimally invasive surgery (robotic-assisted thoracoscopic

Accepted for publication Jun 3, 2020.

Address correspondence to Dr Long, Department of Surgery, UNC School of Medicine, 3040 Burnett-Womack Bldg, CB \#7065, Chapel Hill, NC 27599-7065; email: jason_long@med.unc.edu. respect to age, race, and comorbidities. There were no significant differences in LOS, complications, 30-day readmission and mortality rates, or patient-reported outcomes. Of the patients with prolonged LOS, $31 \%$ had pulmonary complications, almost half of which were prolonged air leaks. ERAS adherence rate was approximately $60 \%$.

Conclusions. In the first year of implementation, median LOS, complications, and 30-day outcomes did not differ significantly between the pre-ERAS and postERAS groups. Prolonged air leaks commonly led to prolonged LOS; therefore, thoracic ERAS protocols could include interventions to reduce air leak and consideration for discharging patients with chest tubes placed to Heimlich valves. Buy-in and adherence to a new protocol are necessary for implementation to be effective.

surgery [RATS] or video-assisted thoracoscopic surgery [VATS]) where possible; and finally, the postoperative phase involves use of incentive spirometry, early mobilization, early chest tube, and urinary catheter removal. ${ }^{2,3}$

Initial thoracic ERAS studies were mostly case series; the lack of a control group increases risk of bias and limits the ability to attribute improvements in care to ERAS. ${ }^{4-6}$ While some recent thoracic ERAS studies were inconclusive, ${ }^{7}$ others showed that ERAS improves outcomes after lung resections and provides more cost-effective care. ${ }^{8,9}$ Objectives of this study were (1) to investigate the effect of ERAS implementation-in comparison with conventional care-on pulmonary resection outcomes at the University of North Carolina (UNC) hospitals; (2) assess the association between demographic, preoperative, anesthesia, and surgical factors on LOS, morbidity, and mortality; and finally, (3) describe challenges of ERAS implementation. 
Table 1. University of North Carolina Thoracic ERAS Protocol

\begin{tabular}{|c|c|c|}
\hline Preoperative & Intraoperative & Postoperative \\
\hline $\begin{array}{l}\text { Tobacco cessation Incentive spirometry } \\
\text { education } \\
\text { Preoperative nutritional screening and } \\
\text { oral carbohydrate loading } \\
\text { Day of surgery: } \\
\text { Preoperative medications: Tylenol } \\
1000 \mathrm{mg} \text { PO, Celebrex } 200 \mathrm{mg} \\
\text { PO, Pregabalin } 100 \mathrm{mg} \text { PO } \\
\text { Consumption of clear fluids up } \\
\text { until } 2 \text { h before surgery. }\end{array}$ & 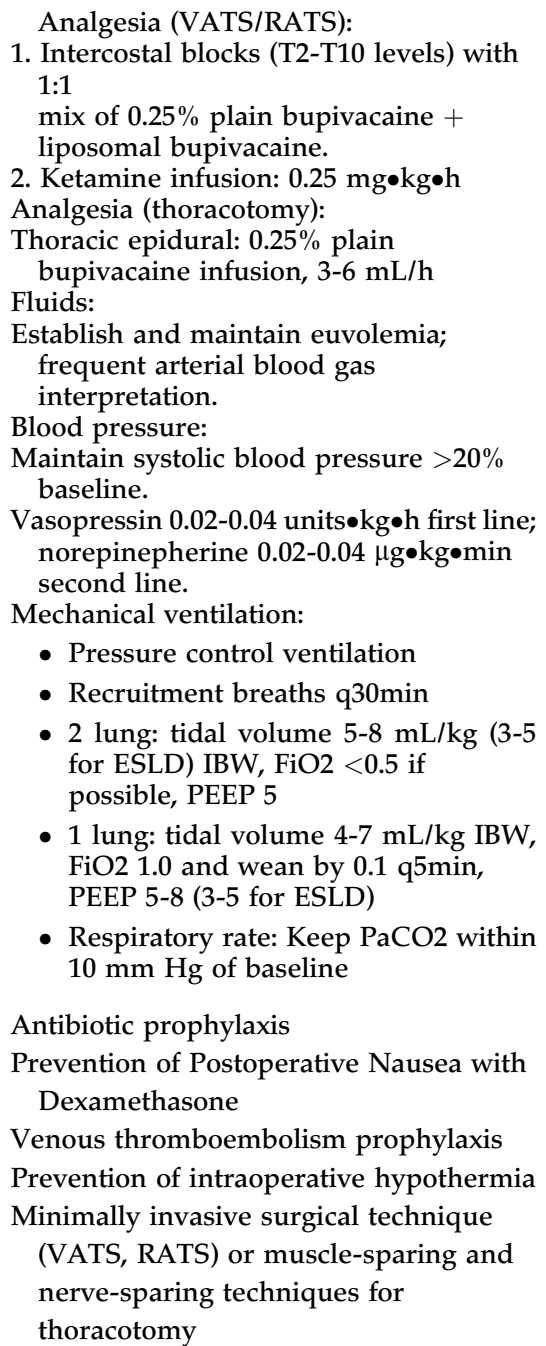 & $\begin{array}{l}\text { Analgesia (VATS/RATS): } \\
\text { - NSAIDs-scheduled } \\
\text { - Ketorolac } 15 \mathrm{mg} \text { IV q8h or ibuprofen } \\
800 \mathrm{mg} \text { PO q8h } \times 24 \mathrm{~h} \text { on POD } 0 \text {. } \\
\text { - Transition to Ibuprofen } 600 \mathrm{mg} \text { PO } \\
\text { q6hr PRN on POD } 1 \text { and until } \\
\text { discharge. } \\
\text { - Gabapentin } 300 \mathrm{mg} \text { PO TID until } \\
\text { discharge. } \\
\text { - Tylenol } 650 \mathrm{mg} \text { PO QID until } \\
\text { discharge. } \\
\text { Early mobilization } \\
\text { Early chest tube removal (24-h } \\
\text { output }<250 \mathrm{~mL} \text { and no air leak) } \\
\text { Early urinary catheter removal }\end{array}$ \\
\hline
\end{tabular}

ERAS, enhanced recovery after surgery; ESLD, end-stage lung disease; $\mathrm{FiO}_{2}$, fraction of inspired oxygen; IBW, ideal body weight; IV, intravenous; NSAID, nonsteroidal antiinflammatory drug; $\mathrm{PaCO}_{2}$, partial pressure of carbon dioxide; PEEP, positive end-expiratory pressure; PO, by mouth; POD, postoperative day; PRN, as needed; q, every; QID, 4 times a day; RATS, robotic-assisted thoracoscopic surgery; TID, 3 times a day; VATS, video-assisted thoracoscopic surgery.

\section{Patients and Methods}

\section{Study Design}

In this retrospective observational study, consecutive adult patients ( $\geq 18$ years of age) undergoing pulmonary resections (wedge resection, segmentectomy, lobectomy, and bilobectomy) during pre-ERAS (April 2014 to September 2015) and post-ERAS (January 2016 to May 2017) periods were identified from UNC's Thoracic Surgery Database. Only a patient's first thoracic operation was eligible; redo surgeries and unexpected returns to the operating room were excluded.

We developed our thoracic ERAS pathway with fundamental elements of previously published protocols. $^{10}$ Evidence-based practices for multimodal opioid-sparing analgesia, including ICB, percutaneous injection of bupivacaine or liposomal bupivacaine under direct vision, as previously described by Van Haren and colleagues ${ }^{11}$; minimally invasive surgery; pulmonary toileting; and chest tube management, were applied to our protocol (Table 1).

Because ERAS was not fully implemented until January 2016, resections performed during this ERAS wash-in period (October to December 2015) were excluded. Patients who simultaneously underwent esophagectomy $(\mathrm{n}=2)$, pneumonectomy $(\mathrm{n}=1)$, or coronary artery bypass grafting $(n=1)$, and trauma patients $(n=4)$ were excluded. Patient demographics, baseline clinical 
Table 2. Patient Baseline Characteristics, Stratified by Pre-ERAS and Post-ERAS Implementation

\begin{tabular}{|c|c|c|c|}
\hline Variable & Pre-ERAS & Post-ERAS & $P$ Value \\
\hline Patients & $133(50)$ & $131(50)$ & \\
\hline Age, y & $64(55-70)$ & $61(54-68)$ & .19 \\
\hline Male & $67(50)$ & $50(38)$ & .05 \\
\hline \multicolumn{4}{|l|}{ Race } \\
\hline White & $100(75)$ & $92(70)$ & .41 \\
\hline Black & $27(20)$ & $28(21)$ & .88 \\
\hline Other & $6(5)$ & $11(8)$ & .22 \\
\hline BMI, $\mathrm{kg} / \mathrm{m}^{2}$ & $27(24-33)$ & $27(23-31)$ & .26 \\
\hline \multicolumn{4}{|l|}{ Comorbidities } \\
\hline Hypertension & $73(55)$ & $60(46)$ & .18 \\
\hline Congestive heart failure & $12(9)$ & $4(3)$ & .07 \\
\hline Coronary artery disease & $20(15)$ & $13(10)$ & .26 \\
\hline Peripheral vascular disease & $13(10)$ & $13(10)$ & .99 \\
\hline Diabetes, insulin-dependent & $6(5)$ & $3(2)$ & .50 \\
\hline Renal insufficiency & $5(4)$ & $6(5)$ & .77 \\
\hline \multicolumn{4}{|l|}{ Smoking status } \\
\hline Never & $35(26)$ & $36(27)$ & .89 \\
\hline Former & $69(52)$ & $72(55)$ & .62 \\
\hline Current & $29(22)$ & $23(18)$ & .44 \\
\hline Smoked within 4 wk of surgery & $34(26)$ & $29(22)$ & .56 \\
\hline Preoperative or longstanding steroid use & $37(28)$ & $64(49)$ & $<.01$ \\
\hline Preoperative chemotherapy & $29(22)$ & $19(15)$ & .15 \\
\hline Preoperative radiation & $27(20)$ & $15(11)$ & .06 \\
\hline Prior cardiothoracic surgery & $15(11)$ & $17(13)$ & .71 \\
\hline \multicolumn{4}{|l|}{ Primary diagnosis } \\
\hline Benign lung disease & $25(19)$ & $24(18)$ & .99 \\
\hline Lung cancer & $77(58)$ & $69(53)$ & .46 \\
\hline Pulmonary metastastectomy & $31(23)$ & $32(24)$ & .89 \\
\hline $\mathrm{FEV}_{1}, \%$ & $83(72-97)$ & $83(66-97)$ & .88 \\
\hline DLCO, \% & $70(57-84)$ & $75(62-87)$ & .16 \\
\hline Karnofsky performance scale index, $\%$ & $100(90-100)$ & $90(90-100)$ & .44 \\
\hline \multicolumn{4}{|l|}{ ASA score } \\
\hline 1 & $0(0)$ & $0(0)$ & $\ldots$ \\
\hline 2 & $13(10)$ & $12(9)$ & .99 \\
\hline 3 & $96(72)$ & $98(75)$ & .68 \\
\hline 4 & $24(18)$ & $21(16)$ & .74 \\
\hline \multicolumn{4}{|l|}{ Thoracic revised cardiac risk index } \\
\hline 0 & $104(78)$ & $1112(86)$ & .15 \\
\hline $1-1.5$ & $25(19)$ & $15(11)$ & .12 \\
\hline$\geq 2$ & $4(3)$ & $4(3)$ & .99 \\
\hline
\end{tabular}

Values are $\mathrm{n}(\%)$ or median (interquartile range).

ASA, American Society of Anesthesiologists; BMI, body mass index; DLCo, diffusing capacity of lung for carbon monoxide; ERAS, enhanced recovery after surgery; $\mathrm{FEV}_{1}$, forced expiratory volume in 1 second.

measures, and surgical characteristics were abstracted from electronic medical records. The Karnofsky performance scale index ${ }^{12}$ and thoracic revised cardiac risk index (ThRCRI) (a validated cardiac risk assessment tool) ${ }^{13}$ were assessed.

Primary outcomes were hospital LOS, total postoperative complications (defined using The Society of Thoracic Surgeons [STS] criteria), ${ }^{14}$ 30-day readmission, and mortality. LOS was categorized as being expected or normal or prolonged based on procedure type, with LOS greater than 2 days after wedge resection, LOS greater than 3 days after segmentectomy, and LOS greater than 5 days after lobe resection or bilobectomy being considered prolonged hospitalizations. ${ }^{15-22}$

\section{Statistical Analyses}

Patient demographics, surgical characteristics, and postoperative outcomes were compared between preERAS and post-ERAS patients using Fisher's exact and Wilcoxon-Mann-Whitney tests, where appropriate. 
Table 3. Intraoperative Characteristics, Stratified by Pre-ERAS and Post-ERAS Implementation

\begin{tabular}{|c|c|c|c|}
\hline Variable & Pre-ERAS $133(50 \%)$ & Post-ERAS $131(50 \%)$ & $P$ Value \\
\hline Patients & $133(50)$ & $131(50)$ & \\
\hline \multicolumn{4}{|l|}{ Preoperative medications } \\
\hline Acetaminophen & 7 (5) & $102(78)$ & $<.0001$ \\
\hline Pregabalin & $5(4)$ & $85(65)$ & $<.0001$ \\
\hline Celecoxib & $1(1)$ & $73(56)$ & $<.0001$ \\
\hline \multicolumn{4}{|l|}{ Procedure } \\
\hline Wedge resection & $85(64)$ & $74(56)$ & .22 \\
\hline Segmentectomy & $0(0)$ & $1(1)$ & .50 \\
\hline Lobectomy & $46(35)$ & $56(43)$ & .21 \\
\hline Bilobectomy & $2(2)$ & $0(0)$ & .50 \\
\hline Robotic surgery & $0(0)$ & $15(11)$ & $<.0001$ \\
\hline Thoracotomy & $3(2)$ & $3(2)$ & .99 \\
\hline Surgery time, $\min$ & $121(67-182)$ & $119(81-193)$ & .34 \\
\hline Anesthesia time, min & $159(113-217)$ & $173(126-232)$ & .18 \\
\hline \multicolumn{4}{|l|}{ Intraoperative medications } \\
\hline Ketorolac & $3(2)$ & $26(20)$ & $<.0001$ \\
\hline Dexamethasone & $44(33)$ & $69(53)$ & .002 \\
\hline Vasopressin & $9(7)$ & $51(39)$ & $<.0001$ \\
\hline Norepinephrine & $1(1)$ & $18(14)$ & $<.0001$ \\
\hline \multicolumn{4}{|l|}{ Regional block type } \\
\hline Intercostal nerve block & $90(68)$ & $102(78)$ & .07 \\
\hline Epidural & $0(0)$ & $0(0)$ & $\ldots$ \\
\hline Intercostal nerve block and epidural & $43(32)$ & $29(22)$ & .07 \\
\hline \multicolumn{4}{|l|}{ Intercostal nerve block type } \\
\hline Bupivacaine alone & $133(100)$ & $37(28)$ & $<.0001$ \\
\hline Liposomal bupivacaine alone & $0(0)$ & $61(47)$ & $<.0001$ \\
\hline Bupivacaine and liposomal bupivacaine & $0(0)$ & $33(25)$ & $<.0001$ \\
\hline Transfusion & $0(0)$ & $0(0)$ & $\ldots$ \\
\hline Total crystalloids, mL & $2000(900-1600)$ & $800(496-1100)$ & $<.0001$ \\
\hline Total colloids, mL & $0(0-0)$ & $250(0-500)$ & $<.0001$ \\
\hline Morphine equivalents, $\mathrm{mg}$ & $25(22-32)$ & $20(15-25)$ & $<.0001$ \\
\hline
\end{tabular}

Values are $\mathrm{n}(\%)$ or median (interquartile range).

ERAS, enhanced recovery after surgery.

A $P$ value less than or equal to .05 was considered statistically significant. Linear regression was used to assess trends in patient-reported satisfaction and pain scores (data from Hospital Consumer Assessment of Healthcare Providers and Systems and Press Ganey).

Cox proportional hazards regression was used to estimate the effect of ERAS on inpatient complications and 30-day readmission. Multivariable hazards regression was used to estimate the effect of ERAS on outcomes, after adjusting for age, gender, steroid use, ThRCRI, intraoperative crystalloids, colloids, and morphine equivalents. Age, crystalloids, colloids, and morphine equivalents were modeled as restricted quadratic splines to allow for the most flexibility. Multivariable logistic regression was used to assess the effect of ERAS on prolonged LOS, after adjusting for variables described previously.

Sensitivity analyses were performed to assess whether potential risk groups were more impacted by ERAS. Specifically, interaction terms were used to assess whether age (categorized as $<70$ and $\geq 70$ years old) and thoracic revised cardiac risk index (categorized as 0 and $\geq 1$ ) modified the effect of ERAS on any complication and prolonged LOS, respectively.

All analyses were performed in SAS version 9.4 (SAS Institute, Cary, NC). The UNC institutional review board provided ethical approval of the study (institutional review board \#15-1841).

\section{Results}

Overall, 264 patients were included; 133 (50\%) underwent surgery pre-ERAS, and $131(50 \%)$ underwent surgery post-ERAS. The groups were comparable in terms of age, race, body mass index, comorbidities, smoking, preoperative chemoradiation, prior cardiothoracic surgery, primary diagnosis, pulmonary function tests, Karnofsky performance scale index, American Society of Anesthesiologists score, or ThRCRI (Table 2). Post-ERAS patients were less likely to be male ( $50 \%$ vs $38 \% ; P=.05)$ and more 
Table 4. Adjusted Effect of ERAS Implementation, Compared With Pre-ERAS Implementation, on Inpatient Complications and 30-Day Outcomes

\begin{tabular}{|c|c|c|c|}
\hline Variable & Pre-ERAS & Post-ERAS & $\operatorname{HR}(95 \% \mathrm{CI})^{\mathrm{a}}$ \\
\hline \multicolumn{4}{|c|}{ Inpatient complications } \\
\hline Pulmonary & $20(15)$ & $20(15)$ & $0.79(0.27-2.34)$ \\
\hline Cardiovascular & $12(9)$ & $9(7)$ & $1.07(0.27-4.20)$ \\
\hline Urinary & $10(8)$ & $17(13)$ & $0.84(0.25-2.88)$ \\
\hline Infection & $2(2)$ & $1(1)$ & NA \\
\hline Hemolytic & $2(2)$ & $2(2)$ & NA \\
\hline Gastrointestinal & $2(2)$ & $0(0)$ & NA \\
\hline Neurological & $5(4)$ & $1(1)$ & NA \\
\hline Other & $4(3)$ & $6(5)$ & $0.93(0.12-7.50)$ \\
\hline Any complication & $36(27)$ & $44(34)$ & $1.25(0.62-2.51)$ \\
\hline 30-d mortality & $0(0)$ & $2(2)$ & NA \\
\hline \multirow[t]{2}{*}{ 30-d readmission } & $11(8)$ & $13(10)$ & $1.85(0.52-6.66)$ \\
\hline & & & OR $(95 \% \mathrm{CI})^{\mathrm{a}}$ \\
\hline Prolonged LOS ${ }^{\mathrm{b}}$ & $65(49)$ & $54(49)$ & $1.55(0.69-3.47)$ \\
\hline
\end{tabular}

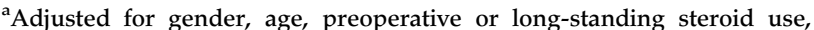
thoracic revised cardiac risk index, intraoperative crystalloids, intraoperative colloids, and intraoperative morphine equivalents; age, crystalloids, colloids, and morphine equivalents were modeled as restricted quadratic splines; $\quad{ }^{b}$ Prolonged LOS was defined as a LOS $>2$ days after wedge resection, $>3$ days after segmentectomy, and $>5$ days after lobe resection or bilobectomy.

Values are $\mathrm{n}(\%)$.

$\mathrm{CI}$, confidence interval; ERAS, enhanced recovery after surgery; HR, hazard ratio; LOS, length of stay; NA, not analyzable; OR, odds ratio.

likely to have preoperative or longstanding steroid use (systemic intravenous or oral steroids), a variable routinely collected by the STS $(28 \%$ vs $49 \% ; P<.01)$. Of note, we initially analyzed steroid use including inhaled steroids and also found a significant difference (35\% vs $56 \% ; P=.001)$.

Post-ERAS patients had higher use of preoperative medications (acetaminophen, pregabalin, and celecoxib), RATS, and intraoperative medications (ketorolac, dexamethasone, vasopressin, and norepinephrine). Cohorts differed significantly in ICB type, total crystalloids and colloids, and morphine equivalents (Table 3 ). No differences were seen across procedure type, surgery or anesthesia time, or regional block type.

Median LOS among all patients was 4 (interquartile range, 2-6) days. Of the 119 patients with prolonged LOS, $37(31 \%)$ had pulmonary complications, almost half of which $(\mathrm{n}=15,41 \%)$ were prolonged air leaks (PALs) $(>5$ days, as defined by the STS). ${ }^{14}$ There were no significant differences in complications, prolonged LOS, 30-day mortality, or readmission between cohorts (Table 4). Even after adjustment, no differences in complications (hazard ratio, 1.25 ; 95\% confidence interval [CI], 0.622.51), 30-day readmission (hazard ratio, 1.85 ; $95 \% \mathrm{CI}, 0.52-$ 6.66 ), or prolonged LOS (odds ratio, 1.55 ; 95\% CI, 0.69 3.47) were seen (Table 4). No difference in the impact of ERAS on complications and prolonged LOS was seen among older $(P=.34$ and $P=.55$, respectively) or highrisk patients $(P=.34$ and $P=.26$, respectively). There were only 2 cases of postoperative mortality (both postERAS). No changes in patient-reported satisfaction or pain management were seen (Figures 1 and 2).

Among patients with prolonged LOS, no difference in PALs was seen across pre-ERAS and post-ERAS implementation $(14 \%$ vs $11 \% ; P=.79)$. Neither low preoperative diffusing capacity for carbon monoxide $(<50 \%)(19 \%$ vs $10 \% ; P=.28)$ nor forced expiratory volume in 1 second (median 85.5 vs $82.3 ; P=.66$ ) was associated with PALs.
Figure 1. Trends in Hospital Consumer Assessment of Healthcare Providers and Systems (HCAHPS) patient satisfaction. Based on HCAHPS data, no changes in patient-reported overall satisfaction or pain management were seen throughout pre-enhanced recovery after surgery and post-enhanced recovery after surgery time periods.

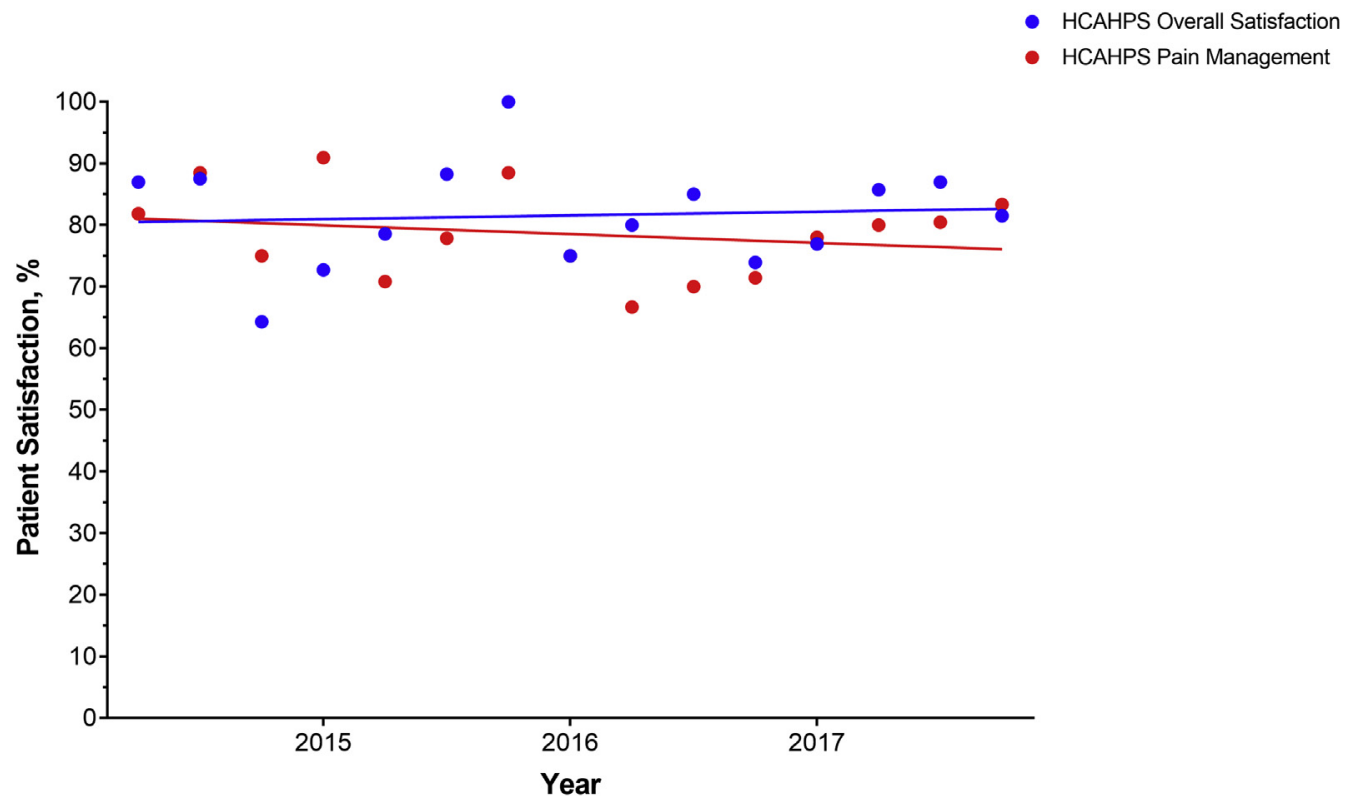




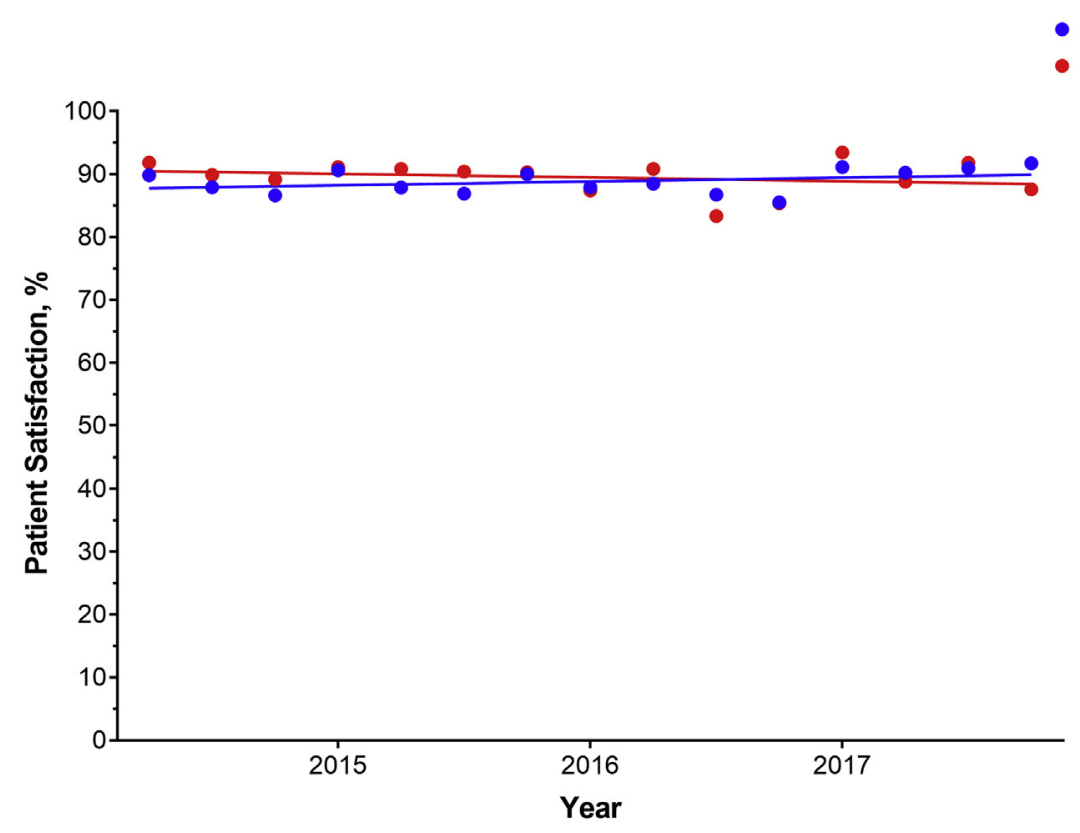

Press Ganey Overall

Press Ganey Pain Control
Figure 2. Trends in mean Press Ganey satisfaction and pain scores based on Press Ganey data. No changes in patient-reported overall satisfaction or pain management were seen throughout pre-enhanced recovery after surgery and post-enhanced recovery time periods.
PALs were not associated with steroid use but were associated with smoking $(P=.03)$.

\section{Comment}

We evaluated preliminary progress in thoracic ERAS implementation at our institution. Pre-ERAS and postERAS patients were comparable preoperatively and only differed in gender and steroid use. Frequent steroid use was an unintended variable that could negatively affect post-ERAS outcomes. ${ }^{23}$ Cohorts differed in intraoperative goal-directed fluid management and multimodal opioid-sparing analgesia, both key ERAS components. Most patients had minimally invasive surgery. Fifteen post-ERAS patients underwent RATS, which is shown to reduce LOS and 30-day mortality when compared with VATS and thoracotomies. ${ }^{24,25}$

Based on preliminary results, median hospital LOS, complications, 30-day readmission and mortality, patientreported satisfaction, and pain did not differ between cohorts. Median LOS was 4 days for both cohorts overall, 3 days for both VATS wedge resection groups, and 5 days for both VATS lobectomy groups; these are comparable with national medians. ${ }^{15-22}$ This was consistent with a Brunelli and colleagues ${ }^{7}$ study that showed no benefit from ERAS on LOS, cardiopulmonary complications, 30day and 90-day mortality, and readmissions. However, this contradicts recent studies that showed decreased LOS and complications and improved patient satisfaction and pain with ERAS. ${ }^{8,9,26}$ One potential reason is that certain ERAS elements (eg, VATS and preoperative counseling) were utilized pre-ERAS. VATS is associated with lower risk and uncomplicated recovery, and thus may not require ERAS. ${ }^{7}$ This concept was demonstrated by Van Haren and colleagues, ${ }^{11}$ who showed decreased
LOS and cardiopulmonary morbidity with ERAS after thoracotomy but not after minimally invasive surgery.

Our data represent a preliminary snapshot of thoracic ERAS implementation at one institution, where preERAS median LOSs were already consistent with national medians. ${ }^{15-22}$ It was difficult to observe significant differences in outcomes given our small sample size and initial low ERAS compliance (certain medications were not always clinically indicated) (Table 3). ERAS implementation is a large-scale quality improvement effort requiring multidisciplinary education and buy-in. We faced many barriers to success, including communication breakdown, staff turnover, lack of resources for data collection and compliance assessment, and the need to update pathways with best-available evidence. ${ }^{27-29}$ Higher ERAS compliance $(>75 \%)$ has been shown to improve outcomes. ${ }^{30}$ Concurrent efforts to improve patient safety and outcomes also render it challenging to determine the overall effect of ERAS.

PALs often extended LOS for our patients. The noted association of PALs with smoking emphasizes the importance of preoperative tobacco cessation. Yoo and colleagues $^{31}$ estimated the economic burden of PAL to be $\$ 6512$ higher. The 2019 Guidelines for Enhanced Recovery After Lung Surgery mention the effect of chest tube suction and digital drainage systems on PALs, ${ }^{32}$ but thoracic ERAS protocols could also include interventions such as biodegradable polymeric lung sealants, ${ }^{33,34}$ buttressing staple lines, ${ }^{35,36}$ or implementation of the Greer and colleagues ${ }^{37}$ Standardized Approach to Air Leak Reduction protocol. Given the steep learning curve of RATS, constant improvement of surgical technique could reduce PAL. ${ }^{38}$ We are also discharging patients with PAL earlier with chest tubes placed to Heimlich valves. ${ }^{39,40}$ 
Finally, $40 \%$ of patients experienced transportationrelated discharge delays. UNC has reduced these delays by providing taxi vouchers and opening a discharge lounge. This phenomenon reveals a potential limitation of using LOS as a metric. Other metrics of ERAS success could include postoperative oxygen requirement and functional recovery metrics (time to return to work or activities of daily living).

Strengths of this study include initial comparability of groups, large number of variables collected, and minimal missing data. However, study limitations include small sample size from one institution and frequent transportation-related discharge delays. Nonresponse bias and memory bias may influence survey pain scores. Lack of a concurrent control group could introduce bias given potential confounding factors. Having VATS as a common element pre-ERAS and post-ERAS may mask the effect of other ERAS elements on outcomes. ${ }^{7}$ Frequent post-ERAS steroid use could impair wound healing and increase infections. ${ }^{23}$ In addition to low ERAS compliance, $^{30}$ the introduction of RATS might also affect postERAS outcomes.

We have discussed initial challenges of thoracic ERAS implementation and strategies to address them. Low compliance, PALs, and discharge delays may offset advantages seen with ERAS. Further studies are warranted as we implement updates from the 2019 guidelines $^{32}$ to improve thoracic ERAS implementation, foster a culture of ongoing quality improvement, and investigate its costeffectiveness.

The authors wish to acknowledge the UNC School of Medicine, UNC Gillings School of Global Public Health, and the Cecil G. Sheps Center for Health Services Research for their collaboration on this project.

\section{References}

1. Melnyk M, Casey RG, Black P, Koupparis AJ. Enhanced recovery after surgery (ERAS) protocols: time to change practice? Can Urol Assoc J. 2011;5:342-348.

2. Gimenez-Mila M, Klein AA, Martinez G. Design and implementation of an enhanced recovery program in thoracic surgery. J Thorac Dis. 2016;8:S37-S45.

3. Rogers LJ, Bleetman D, Messenger DE, et al. The impact of enhanced recovery after surgery (ERAS) protocol compliance on morbidity from resection for primary lung cancer. J Thorac Cardiovasc Surg. 2018;155:1843-1852.

4. Bryant AS, Cerfolio RJ. The analysis of a prospective surgical database improves postoperative fast-tracking algorithms after pulmonary resection. J Thorac Cardiovasc Surg. 2009;137: 1173-1179.

5. Cerfolio RJ, Pickens A, Bass C, Katholi C. Fast-tracking pulmonary resections. J Thorac Cardiovasc Surg. 2001;122:318-324.

6. McKenna RJ Jr, Mahtabifard A, Pickens A, Kusuanco D, Fuller CB. Fast-tracking after video-assisted thoracoscopic surgery lobectomy, segmentectomy, and pneumonectomy. Ann Thorac Surg. 2007;84:1663-1667 [discussion: 1667-1668].

7. Brunelli A, Thomas C, Dinesh P, Lumb A. Enhanced recovery pathway versus standard care in patients undergoing video-assisted thoracoscopic lobectomy. J Thorac Cardiovasc Surg. 2017;154:2084-2090.
8. Madani A, Fiore JF Jr, Wang Y, et al. An enhanced recovery pathway reduces duration of stay and complications after open pulmonary lobectomy. Surgery. 2015;158:899-908 [discussion: 908-910].

9. Paci P, Madani A, Lee L, et al. Economic impact of an enhanced recovery pathway for lung resection. Ann Thorac Surg. 2017;104:950-957.

10. Kolarczyk LM, McNaull P, Hance L, Bednar P, Kim HJ, Isaak R. Implementation of an enhanced recovery after surgery (ERAS) clinical pathway using Lean Six Sigma principles: a framework for ongoing quality improvement. J Anesth Surg. 2016;1:18-22.

11. Van Haren RM, Mehran RJ, Mena GE, et al. Enhanced recovery decreases pulmonary and cardiac complications after thoracotomy for lung cancer. Ann Thorac Surg. 2018;106:272279 .

12. Karnofsky DA, Burchenal JH. The clinical evaluation of chemotherapeutic agents in cancer. In: Macleod CM, ed. Evaluation of Chemotherapeutic Agents. New York, NY: Columbia University Press; 1949:191-205.

13. Thomas DC, Blasberg JD, Arnold BN, et al. Validating the Thoracic Revised Cardiac Risk Index following lung resection. Ann Thorac Surg. 2017;104:389-394.

14. STS National Database. General Thoracic Surgery Database Training Manual, Version 2.41. Chicago, IL: Society of Thoracic Surgeons; September 19, 2018.

15. Seder CW, Salati M, Kozower BD, et al. Variation in pulmonary resection practices between the Society of Thoracic Surgeons and the European Society of Thoracic Surgeons General Thoracic Surgery Databases. Ann Thorac Surg. 2016;101:2077-2084.

16. Dickinson KJ, Taswell JB, Allen MS, et al. Unplanned readmission after lung resection: complete follow-up in a 1-year cohort with identification of associated risk factors. Ann Thorac Surg. 2017;103:1084-1091.

17. Yang CF, D'Amico TA. Thoracoscopic segmentectomy for lung cancer. Ann Thorac Surg. 2012;94:668-681.

18. Rosen JE, Hancock JG, Kim AW, Detterbeck FC, Boffa DJ. Predictors of mortality after surgical management of lung cancer in the National Cancer Database. Ann Thorac Surg. 2014;98:1953-1960.

19. Kozower BD, O'Brien SM, Kosinski AS, et al. The Society of Thoracic Surgeons Composite Score for Rating Program performance for lobectomy for lung cancer. Ann Thorac Surg. 2016;101:1379-1386 [discussion: 1386-1387].

20. Seder CW, Wright CD, Chang AC, Han JM, McDonald D, Kozower BD. The Society of Thoracic Surgeons General Thoracic Surgery Database Update on Outcomes and Quality. Ann Thorac Surg. 2016;101:1646-1654.

21. Kozower BD, Sheng S, O’Brien SM, et al. STS database risk models: predictors of mortality and major morbidity for lung cancer resection. Ann Thorac Surg. 2010;90:875881.

22. Boffa DJ, Allen MS, Grab JD, Gaissert HA, Harpole DH, Wright CD. Data from the Society of Thoracic Surgeons General Thoracic Surgery database: the surgical management of primary lung tumors. I Thorac Cardiovasc Surg. 2008;135:247-254.

23. Ismael $\mathrm{H}$, Horst $\mathrm{M}$, Farooq $\mathrm{M}$, Jordon J, Patton $\mathrm{JH}$, Rubinfeld IS. Adverse effects of preoperative steroid use on surgical outcomes. Am J Surg. 2011;201:305-308.

24. Reddy RM, Gorrepati ML, Oh DS, Mehendale S, Reed MF. Robotic-assisted versus thoracoscopic lobectomy outcomes from high-volume thoracic surgeons. Ann Thorac Surg. 2018;106:902-908.

25. Park BJ, Flores RM. Cost comparison of robotic, videoassisted thoracic surgery and thoracotomy approaches to pulmonary lobectomy. Thorac Surg Clin. 2008;18:297300.

26. Horattas MC, Horattas SA, Heib N, Brandstetter S, Bahr K, Reissig J. Enhanced recovery after surgery results in enhanced patient satisfaction. J Am Coll Surg. 2017;225:e67e68. 
27. Pearsall EA, Meghji Z, Pitzul KB, et al. A qualitative study to understand the barriers and enablers in implementing an enhanced recovery after surgery program. Ann Surg. 2015;261:92-96.

28. McLeod RS, Aarts MA, Chung F, et al. Development of an enhanced recovery after surgery guideline and implementation strategy based on the knowledge-to-action cycle. Ann Surg. 2015;262:1016-1025.

29. Tezber K, Aviles C, Eller M, et al. Implementing enhanced recovery after surgery (ERAS) program on a specialty nursing unit. J Nurs Adm. 2018;48:303-309.

30. Forster C, Doucet V, Perentes JY, et al. Impact of compliance with components of an ERAS pathway on the outcomes of anatomic VATS pulmonary resections. J Cardiothorac Vasc Anesth. 2020;34:1858-1866.

31. Yoo A, Ghosh SK, Danker W, Kassis E, Kalsekar I. Burden of air leak complications in thoracic surgery estimated using a national hospital billing database. Clinicoecon Outcomes Res. 2017;9:373-383.

32. Batchelor TJP, Rasburn NJ, Abdelnour-Berchtold E, et al. Guidelines for enhanced recovery after lung surgery: recommendations of the Enhanced Recovery After Surgery (ERAS(R)) Society and the European Society of Thoracic Surgeons (ESTS). Eur J Cardiothorac Surg. 2019;55:91-115.

33. Allen MS, Wood DE, Hawkinson RW, et al. Prospective randomized study evaluating a biodegradable polymeric sealant for sealing intraoperative air leaks that occur during pulmonary resection. Ann Thorac Surg. 2004;77:1792-1801.

34. Malapert G, Hanna HA, Pages PB, Bernard A. Surgical sealant for the prevention of prolonged air leak after lung resection: meta-analysis. Ann Thorac Surg. 2010;90: 1779-1785.

35. Mery CM, Shafi BM, Binyamin G, Morton JM, Gertner M. Profiling surgical staplers: effect of staple height, buttress, and overlap on staple line failure. Surg Obes Relat Dis. 2008;4: 416-422.

36. Baysungur V, Tezel C, Ergene G, et al. The autologous pleural buttressing of staple lines in surgery for bullous lung disease. Eur I Cardiothorac Surg. 2010;38:679-682.

37. Greer S, Miller AD, Smith JS, Holcombe JM, Headrick JR Jr. Safety of next day discharge after lobectomy: have we broken the speed limit? Ann Thorac Surg. 2018;106:998-1001.

38. Cheufou DH, Mardanzai K, Ploenes T, et al. Effectiveness of robotic lobectomy-outcome and learning curve in a high volume center. Thorac Cardiovasc Surg. 2019;67: 573-577.

39. Varela G, Jimenez MF, Novoa N. Portable chest drainage systems and outpatient chest tube management. Thorac Surg Clin. 2010;20:421-426.

40. Cerfolio RJ, Bass CS, Pask AH, Katholi CR. Predictors and treatment of persistent air leaks. Ann Thorac Surg. 2002;73: 1727-1730 [discussion: 1730-1731]. 